\title{
Partially-coherent spirally-polarized gradual-edge imaging
}

\author{
Marcos Pérez-Aviñoa $^{\mathrm{a}}$, Rosario Martínez-Herrero ${ }^{\mathrm{b}}$, Santiago Vallmitjana ${ }^{\mathrm{a}}$, \\ Pedro Latorre-Carmona ${ }^{\mathrm{c}}$, Ignasi Juvells ${ }^{\mathrm{a}}$, Artur Carnicer ${ }^{*}, \mathrm{a}$ \\ *Corresponding author E-mail: artur.carnicer@ub.edu \\ ${ }^{a}$ Universitat de Barcelona (UB), Facultat de Fúsica, Departament de Física Aplicada, Martí \\ $i$ Franquès 1, 08028 Barcelona, Spain \\ ${ }^{b}$ Universidad Complutense de Madrid, Facultad de Ciencias Físicas, Departamento de \\ Óptica, Ciudad Universitaria, 28040 Madrid, Spain \\ ${ }^{c}$ Universitat Jaume I, Institute of New Imaging Technologies, Campus Riu Sec, 12071 \\ Castelló de la Plana, Spain
}

\begin{abstract}
In this work we develop a partially-coherent spirally-polarized imaging system for generating gradual edge detection images. A rotating diffuser is used for producing illumination sources with tunable degree of coherence. Using a $4 \mathrm{f}$ configuration, the frequency content of the image is modified by means of a vortex half-wave retarder as a spatial filter. Accordingly, we analytically describe a spirally-polarized imaging system illuminated with a Gauss-Schell source using paraxial coherence-polarization theory. Numerical simulations and experimental results demonstrate the validity of our approach. We use a referenceless spatial content descriptor to assess the quality of the recorded images as a function of the coherence state of the source.
\end{abstract}

Keywords: Fourier optics, Spatial filtering, Coherence, Polarization

\section{Introduction}

The theory that describes the statistical properties of electromagnetic fields allows for a precise characterization of coherent phenomena [1-3]. This framework was extended to jointly describe coherence and polarization using a unified 5 vector formalism [4-6]. Moreover, a generalization to partially coherent focused beams was introduced in $[7,8]$. On the other hand, the development of partially coherent sources with tunable degree of coherence is a topic of remarkable interest when dealing with experimental setups [9-13]. Very recently, some authors have proposed spatial filtering systems for producing beams with a given degree 10 of coherence $[14,15]$.

Vortex half-wave retarders (VHR) are useful for producing light beams with radial, azimuthal or spiral polarization and also as a way of enhancing the edges of the image $[16,17]$. Similar results are also obtained by means of spiral phase 
plates, as in [18]. In the present work we demonstrate the feasibility of producing tunable gradual edge detection on the recorded image by means of a partiallycoherent spirally-polarized $4 \mathrm{f}$ imaging system. Interestingly, we found that the partial edge enhancement effect results in an improvement of the spatial quality content of the processed images. To the best of our knowledge, this is the first time this approach is described.

The paper is organized as follows: in section 2 , we mathematically describe a $4 \mathrm{f}$ processing system with a VHR filter placed at the Fourier plane and illuminated with a Gauss-Schell model source. Then, we present an experiment that demonstrates the results predicted by our theoretical approach. In section 4, we discuss the behavior of the recorded images by means of the analysis of the im-

25 age histograms and the use of the Blind/Referenceless Image Quality Evaluator (BRISQUE) [19]. The use of this descriptor is very appropriate because it does not require any perfect reference to assess the quality of the image. Finally, we present our conclusions.

\section{Mathematical description of a partially coherent, radially polar- ized $4 \mathrm{f}$ imaging system}

Figure 1 depicts a $4 \mathrm{f}$ optical system and the variables used in the present analysis. The object $g\left(x_{0}, y_{0}\right)$ is placed at the front focal plane of lens $L_{1}$ and illuminated with a totally polarized beam $u\left(x_{0}, y_{0}\right) \vec{E}_{0}$. Beam $\vec{E}_{0}$ is linearly polarized i.e. $\vec{E}_{0}=(\cos \beta, \sin \beta)$. Coordinate systems $\left(x_{0}, y_{0}\right),\left(x^{\prime}, y^{\prime}\right)$ and $(x, y)$

35 refer to the input, Fourier and output planes, respectively. Polar coordinates $\left(r^{\prime}, \varphi^{\prime}\right)$ (Fourier plane) and $(r, \varphi)$ (output plane) are also used. In what follows, $u\left(x_{0}, y_{0}\right)$ is assumed to be a stochastic process, being $\Gamma\left(x_{1}, y_{1}, x_{2}, y_{2}\right)=$ $\left\langle u^{*}\left(x_{1}, y_{1}\right) u\left(x_{2}, y_{2}\right)\right\rangle$ the mutual coherence function.

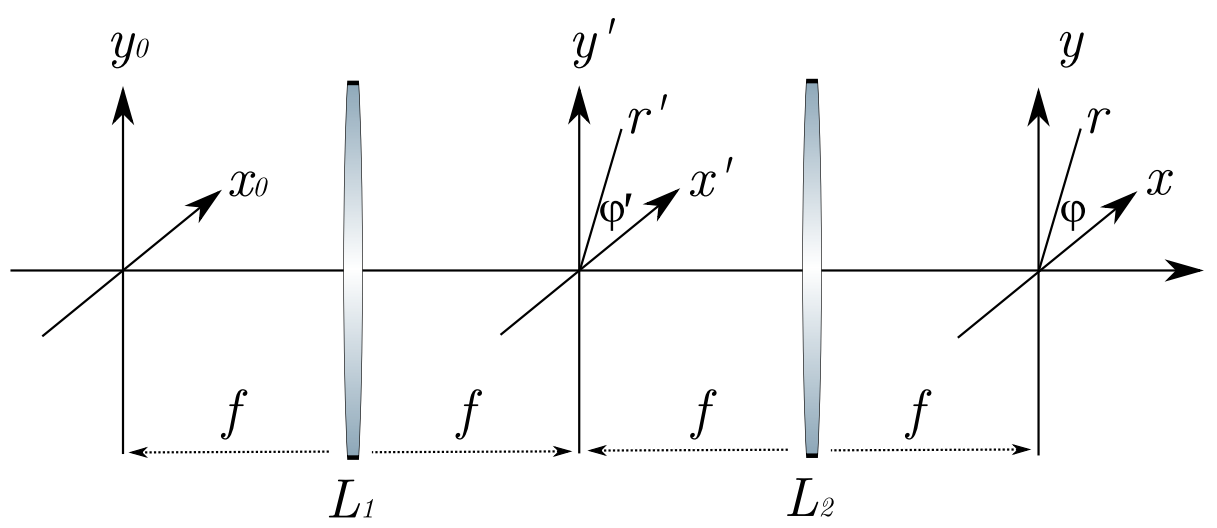

Figure 1: 4f setup 
A VHR filter $\hat{M}$ is set at the back focal plane of $L_{1}$ (Fourier plane). The Jones matrix of such a device is described by

$$
\hat{M}=\left(\begin{array}{cc}
\cos \varphi^{\prime} & \sin \varphi^{\prime} \\
-\sin \varphi^{\prime} & \cos \varphi^{\prime}
\end{array}\right) .
$$

The optical system is described by using paraxial propagation theory [20] and accordingly, the electric field at the back focal plane of tube lens $L_{2}$ (output plane) reads:

$$
\vec{E}(x, y) \propto \iint g(\bar{x}-x, \bar{y}-y) u(\bar{x}-x, \bar{y}-y) \vec{E}_{0} \hat{N}(\bar{x}, \bar{y}) d \bar{x} d \bar{y}
$$

where matrix $\hat{N}$ is the optical Fourier transform of $\hat{M}$, i.e.:

$$
\hat{N}(r, \varphi)=\int \hat{M}\left(r^{\prime}, \varphi^{\prime}\right) \exp \left(i \frac{2 \pi}{\lambda f}\left(r^{\prime} r \cos \left(\varphi^{\prime}-\varphi\right)\right) r^{\prime} d r^{\prime} d \varphi^{\prime} .\right.
$$

Using the previous equation, it is straightforward to demonstrate that matrix $\hat{N}$ reads

$$
\hat{N}(r, \varphi)=2 \pi i\left(\begin{array}{cc}
\cos \varphi & \sin \varphi \\
-\sin \varphi & \cos \varphi
\end{array}\right) \int_{0}^{R} \mathrm{~J}_{1}\left(\frac{2 \pi r^{\prime} r}{\lambda f}\right) r^{\prime} d r^{\prime}
$$

where $R$ is the radius of filter $\hat{M}$ and $J_{1}(x)$ is the Bessel function of the first kind and order 1 . Interestingly, depending on the polarization direction of the input beam $\vec{E}_{0}=(\cos \beta, \sin \beta)$, the electric field at the back focal plane $\vec{E}(x, y)$ is spirally polarized. Then, the recorded intensity $I(x, y)$ is described by

$$
\begin{aligned}
I(x, y) \propto & \int g\left(x_{1}-x, y_{1}-y\right)^{*} g\left(x_{2}-x, y_{2}-y\right) \Gamma\left(x_{1}-x, y_{1}-y, x_{2}-x, y_{2}-y\right) \\
& \operatorname{Tr}\left[\hat{N}^{\dagger}\left(x_{1}, y_{1}\right) \vec{E}_{0}^{\dagger} \vec{E}_{0} \hat{N}\left(x_{2}, y_{2}\right)\right] d x_{1} d y_{1} d x_{2} d y_{2} .
\end{aligned}
$$

Symbol $\dagger$ and operator $\operatorname{Tr}[]$ stand for conjugate transpose matrix and matrix trace respectively; $x_{1}, y_{1}, x_{2}$ and $y_{2}$ are dummy variables. In particular, for linearly polarized input beams $\vec{E}_{0}=(\cos \beta, \sin \beta)$ the trace term reads:

$$
\begin{aligned}
\operatorname{Tr}\left[\hat{N}^{\dagger}\left(x_{1}, y_{1}\right) \vec{E}_{0}^{\dagger} \vec{E}_{0} \hat{N}\left(x_{2}, y_{2}\right)\right]= & 4 \pi^{2} \cos \left(\varphi_{1}-\varphi_{2}\right) \int_{0}^{R} \mathrm{~J}_{1}\left(\frac{2 \pi r^{\prime} r_{1}}{\lambda f}\right) r^{\prime} d r^{\prime} \\
& \times \int_{0}^{R} \mathrm{~J}_{1}\left(\frac{2 \pi r^{\prime} r_{2}}{\lambda f}\right) r^{\prime} d r^{\prime},
\end{aligned}
$$

where $\varphi_{1}$ and $\varphi_{2}$ are the dummy angular coordinates related to $x_{1}, y_{1}$ and $x_{2}$ and $y_{2}$, respectively. For a partially coherent source that fulfills the Gauss-Schell model [2], the mutual coherence function $\Gamma($ ) reads

$\Gamma\left(x_{1}, y_{1}, x_{2}, y_{2}\right)=C \exp \left(-\frac{x_{1}^{2}+x_{2}^{2}+y_{1}^{2}+y_{2}^{2}}{4 \sigma^{2}}\right) \exp \left(-\frac{\left(x_{1}-x_{2}\right)^{2}+\left(y_{1}-y_{2}\right)^{2}}{2 \mu^{2}}\right)$, 
where $C$ is a constant and $\sigma$ and $\mu$ describe the spatial width of the source and the longitude of coherence, respectively. In particular, special cases $\mu / \sigma \longrightarrow 0$ and $\mu / \sigma \longrightarrow \infty$ describe totally incoherent and totally coherent sources respectively. For an incoherent source, the mutual coherence function fulfills $\Gamma\left(x_{1}, y_{1}, x_{2}, y_{2}\right)=\delta\left(x_{1}-x_{2}\right) \delta\left(y_{1}-y_{2}\right)$ and thus, the intensity $I(x, y)$ becomes

$$
\begin{array}{r}
I(x, y)=\int g\left(x_{1}-x, y_{1}-y\right)^{*} g\left(x_{1}-x, y_{1}-y\right) \\
\operatorname{Tr}\left[\hat{N}^{\dagger}\left(x_{1}, y_{1}\right) \vec{E}_{0}^{\dagger} \vec{E}_{0} \hat{N}\left(x_{1}, y_{1}\right)\right] d x_{1} d y_{1}= \\
\int\left|g\left(x_{1}-x, y_{1}-y\right)\right|^{2} \operatorname{Tr}\left[\hat{N}^{\dagger}\left(x_{1}, y_{1}\right) \vec{E}_{0}^{\dagger} \vec{E}_{0} \hat{N}\left(x_{1}, y_{1}\right)\right] d x_{1} d y_{1} .
\end{array}
$$

Note that $h_{i}(x, y)=\operatorname{Tr}\left[\hat{N}^{\dagger}(x, y) \vec{E}_{0}^{\dagger} \vec{E}_{0} \hat{N}(x, y)\right]$ is the incoherent point spread function of the system.

For a full coherent source, the mutual coherence function is simply $\Gamma\left(x_{1}, y_{1}, x_{2}, y_{2}\right)=$ $u^{*}\left(x_{1}, y_{1}\right) u\left(x_{2}, y_{2}\right)$ and consequently, the intensity reads

$$
\begin{aligned}
I(x, y) \propto & \int g^{*}\left(x_{1}-x, y_{1}-y\right) u^{*}\left(x_{1}, y_{1}\right) g\left(x_{2}-x, y_{2}-y\right) u\left(x_{2}, y_{2}\right) \\
& \operatorname{Tr}\left[\hat{N}^{\dagger}\left(x_{1}, y_{1}\right) \vec{E}_{0}^{\dagger} \vec{E}_{0} \hat{N}\left(x_{2}, y_{2}\right)\right] d x_{1} d y_{1} d x_{2} d y_{2}= \\
& \left|\int g^{*}(\bar{x}-x, \bar{y}-y) u^{*}(\bar{x}, \bar{y}) \vec{E}_{0} \hat{N}(\bar{x}, \bar{y}) d \bar{x} d \bar{y}\right|^{2} .
\end{aligned}
$$

In order to provide more insight into the behavior of the described system, we calculated the intensity $I(x, y)$ using Eq. (5) for different values of parameter $\mu$ (Eq. (7)); the intensity over the test object is nearly constant. The test object $g(x, y)$ is a 201x201 pixels image shown in Fig. 2(a); the length of the image

45 width is set to $20 \mathrm{~mm}$ (the radius of the star is $10 \mathrm{~mm}$ ) and $\vec{E}_{0}$ is linearly polarized. Figures 2(b-f) display the resulting distributions $I(x, y)$ for $\mu=10^{6}$, 100, 10, 3 and $0.1 \mathrm{~mm}$ respectively.

Figure 2(b) shows the resulting image for $\mu=10^{6}$; the VHR filter produces an edge enhancement effect, removing all light but from the edges. Moreover, the 50 fine details at the center of the star are lost due to the coherent cut-off frequency. Figures $2(\mathrm{c}-\mathrm{d})$ were calculated using $\mu=100$ and $\mu=10$ respectively; note that Figs. 2(b-d) are almost indistinguishable. On the other hand, Fig. 2(f) displays the most incoherent case considered in this set of simulations $(\mu=0.1$ $\mathrm{mm})$. As expected, the coherent cut-off frequency limit is over-passed at the 55 expense of a contrast inversion effect [20]; moreover, no edge enhancement is reported. Finally, Fig. 2(e) $(\mu=3 \mathrm{~mm})$ shows an intermediate state: edge enhancement is present, but some light is detectable in the pixels that do not belong to the edges of the original image. According to these results, it would be possible to produce gradual edge-enhancement images by tuning the coherence ${ }_{60}$ of the source. Interestingly, changes in the behavior of the final image are only noticeable for $\mu$ values small enough so that the illumination source can be considered nearly incoherent. 


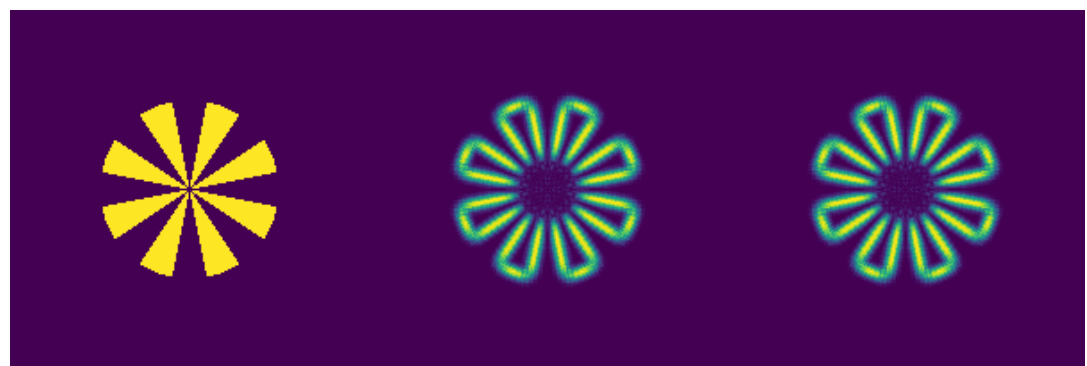

(a)

(b)

(c)

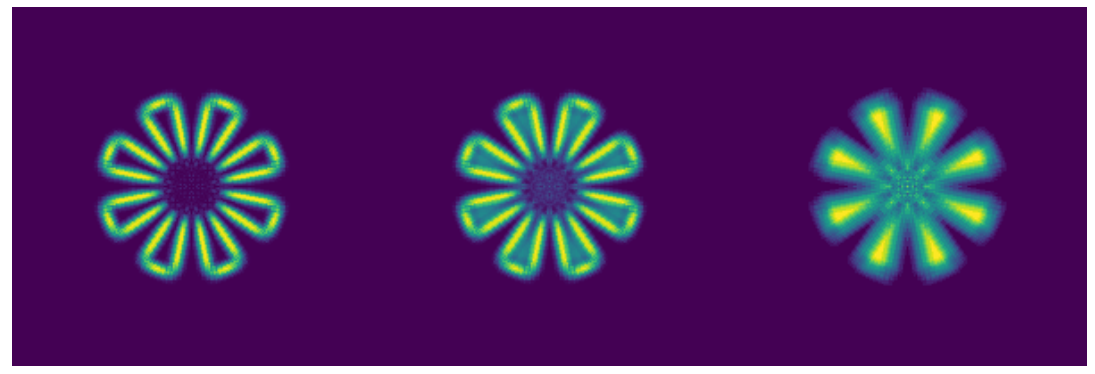

(d)

(e)

(f)

Figure 2: Image formation simulations using partially coherent light. Images are presented in false color using the viridis colormap: (a) test object; (b) $I(x, y)$ with $\mu=10^{6} \mathrm{~mm}$; (c) $I(x, y)$ with $\mu=100 \mathrm{~mm}$; (d) $I(x, y)$ with $\mu=10 \mathrm{~mm}$; (e) $I(x, y)$ with $\mu=3 \mathrm{~mm}$; (f) $I(x, y)$ with $\mu=0.1 \mathrm{~mm}$.

\section{Experimental set-up}

In order to test the theoretical approach described above, an optical experiment has been conducted. Figure 3 shows a sketch of the optical setup and a picture of the system. The sample $g(x, y)$ is imaged by means of a bench microscope comprised of a $\mathrm{NA}=0.45$ microscope lens (ML), a tube lens (TL) and a CCD camera. Object $g(x, y)$ and a VHR filter (Thorlabs WPV10L-633) are set at the front and back focal planes of the objective lens respectively. Since

70 $f_{\mathrm{ML}} \neq f_{\mathrm{TL}}$, coordinates $(x, y)$ at the CCD plane have to be scaled according to a magnification factor $M=-\frac{f_{\mathrm{TL}}}{f_{\mathrm{ML}}}$. The sample is illuminated by means of a condenser system able to tune the degree of coherence of the beam.

A He-Ne laser $(\lambda=632.8 \mathrm{~nm})$ connected to an optical fiber produces a diverging wave located at point $S$. The beam is propagated through lenses $\mathrm{L}_{A}, \mathrm{~L}_{B}$ 75 and $\mathrm{L}_{C} ; S^{\prime}$ and $S^{\prime \prime}$ are intermediate images of the point source $S$ after lenses $\mathrm{L}_{A}$ and $\mathrm{L}_{C}$ respectively. A rotating diffuser is placed between lenses $\mathrm{L}_{A}$ and $\mathrm{L}_{B}$; the position of the diffuser can be modified in order to change the coherence of the light that illuminates the sample, being $d$ the distance between $S^{\prime}$ and the difusser. As we show below, $d$ is a handy parameter to characterize the degree 
of coherence of the beam.

The coherence length $\mu$ at the sample plane has been theoretically estimated using the VanCitter-Zernike theorem, and taking into account the specifications of the condenser system. As shown in [21] and in [5] (pages 31 and 32), in an experimental setup used for producing a Gauss-Schell source, parameters $\sigma$ and $\mu$ are related by means of he following equation

$$
\mu=\frac{D \lambda}{\sqrt{2} \pi \sigma},
$$

where $D$ is the distance between the rotating diffuser and the sample.

We evaluated the effective coherence length on the sample $\mu^{\prime}(d)$ for the present optical setup. First, the positions $D^{\prime}(d)$ of the image of the difusser through lenses $\mathrm{L}_{B}$ and $\mathrm{L}_{C}$ were calculated. Second, rough estimations of the

85 beam widths $\sigma(d)$ at the difusser plane were obtained; then, effective widths on the sample $\sigma^{\prime}(d)$ were determined by considering the magnification through the optical system. Finally, $\mu^{\prime}(d)$ was assessed by using the effective parameters $D^{\prime}(d)$ and $\sigma^{\prime}(d)$ on Eq. 10. Finally, we estimated that $\mu$ values varies from 0.14 to $0.0038 \mathrm{~mm}$ for $0.7 \leq d \leq 25 \mathrm{~mm}$. The specifications of the optical system are 90 summarized in Table 1.
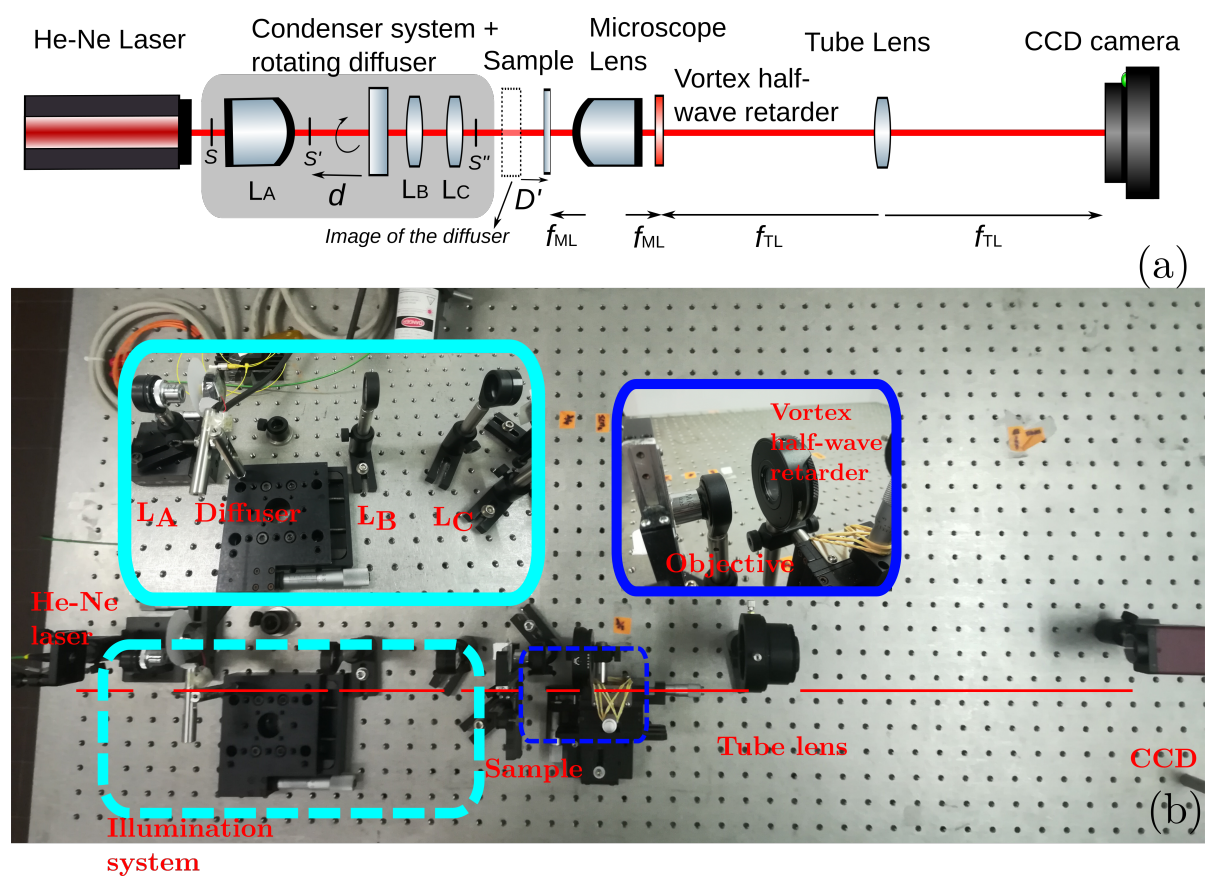

Figure 3: (a) Sketch of the optical setup; (b) experimental optical arrangement 


\begin{tabular}{|l|l|}
\hline Lens $\mathrm{L}_{A}$ & $f_{A}=13 \mathrm{~mm}, \mathrm{NA}=0.45$ \\
Lens $\mathrm{L}_{B}$ & $f_{B}=150 \mathrm{~mm}$ \\
Lens $\mathrm{L}_{C}$ & $f_{C}=25 \mathrm{~mm}$ \\
Microscope Lens (ML) & $f_{\mathrm{ML}}=16 \mathrm{~mm}, \mathrm{NA}=0.45$ \\
Tube Lens (TL) & $f_{\mathrm{TL}}=200 \mathrm{~mm}$ \\
\hline Distance $\mathrm{L}_{A}-\mathrm{S}$ & $\approx 25 \mathrm{~mm}$ \\
${\text { Distance } \mathrm{L}_{A}-\mathrm{L}_{B}}$ & $170 \mathrm{~mm}$ \\
Distance $\mathrm{L}_{B}-\mathrm{L}_{C}$ & $115 \mathrm{~mm}$ \\
Distance $\mathrm{L}_{C}-$ Sample & $54 \mathrm{~mm}$ \\
Range distance $d$ & $0.7-25.5 \mathrm{~mm}$ \\
\hline Source wavelength & $632.8 \mathrm{~nm}$ \\
Object area & $0.64 \times 0.44 \mathrm{~mm}$ \\
$\#$ pixels & $1280 \times 960$ \\
Bit depth & 8 \\
\hline
\end{tabular}

Table 1: Specifications of the optical system.

\section{Results}

Three objects have been used: a USAF-1951 frequency test and two different pictures of diatoms. These images have been processed with and without the VHR filter. For each of the six cases considered, twenty different intensity 95 images have been recorded at different distances $d$. Four images of each set recorded at $d=0.7,5.23,13.8$ and $25.5 \mathrm{~mm}$ are shown in Fig. $4(\mu=0.14,0.018$, $0.007,0.0038 \mathrm{~mm}$ respectively). The images in rows 1,3 and 5 were obtained without the VHR filter; images displayed in rows 2, 4 and 6 were acquired using the VHR filter.

In the images of the first column, high coherence noise is noticeable and in those where the polarizing filter is present a clear edge enhancement effect is detected. The second and third columns show a reduction of the coherent noise; when the VHR filter is used the background intensity increases. In the last column, all coherent effects, including edge enhancement, disappear. The final images, with and without the filter, are very similar.

An interesting behavior is found when analyzing the histograms of the images [Fig. 5]. Note that when the filter is used the histograms are shifted towards lower pixel values as the coherence of the light increases. This is consistent with Fig. 4: whereas filtered images illuminated with partially coherent light contain almost only black pixels, almost incoherent light produces images with a more energetic background.

Although informative, histograms do not provide any clue about the quality of the processed images. In the present work, we are interested in the assessment of the quality of the recorded images, since we are aiming at determining

115 under which conditions we get the best results. One of the most accepted blind image quality estimators is BRISQUE. This approach is chosen because there is no reference image among the recorded ones whose quality can be considered as perfect. This method builds local spatial features for images (images 


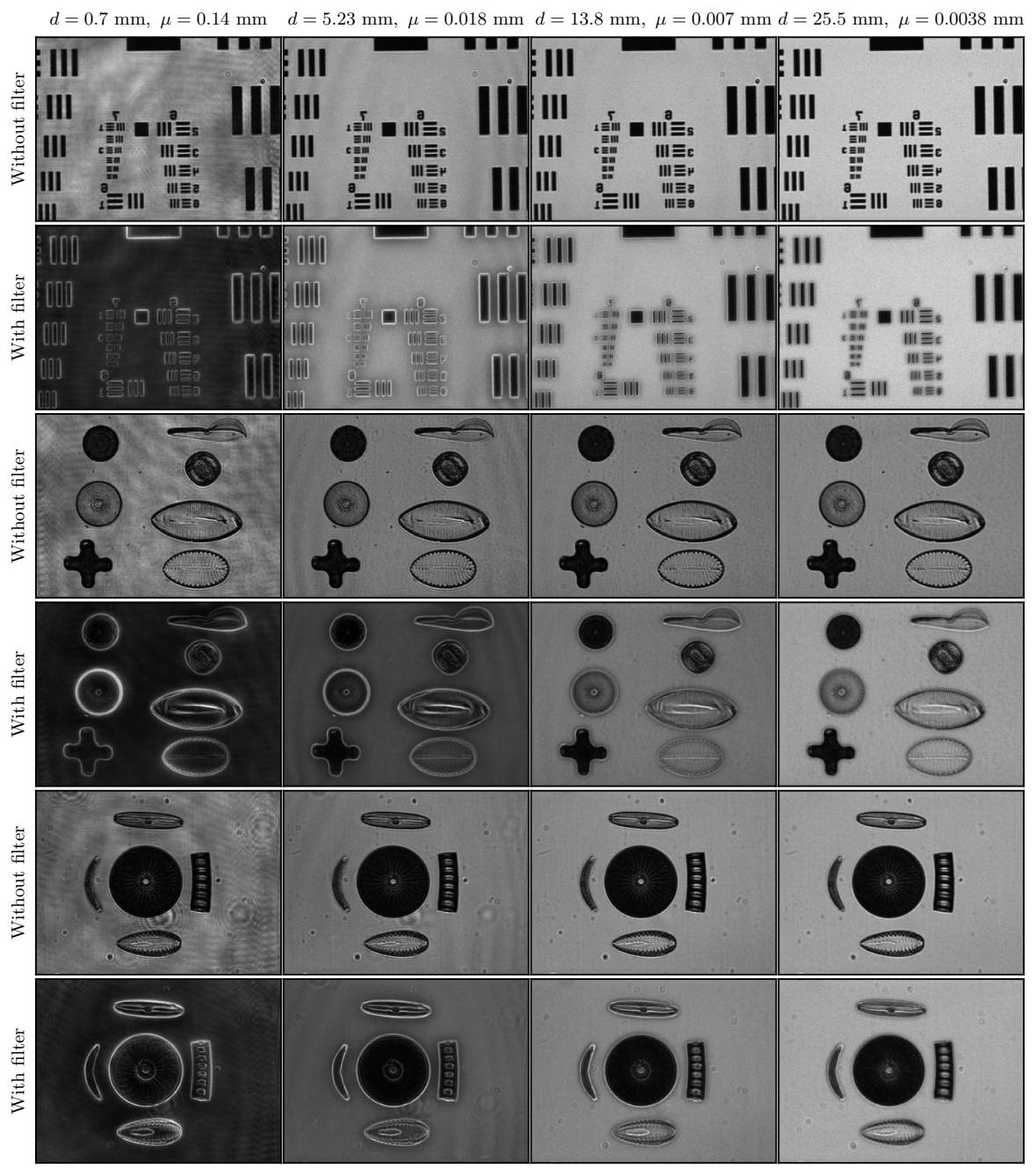

Figure 4: Recorded images $I(x, y)$ at different distances $d$ with their corresponding coherence length $\mu$. The results shown in the first, third and fifth rows do not use the VHR filter. The second, fourth and, sixth rows correspond to the images recorded with the filter.

patches of size typically $7 \times 7$ ) called subtracted contrast normalized coeffi120 cients and hypothesize on the fact that these coefficients have characteristic statistical properties that are changed under the presence of a distortion. In particular, authors in [19] model these coefficients when no distortion appears as asymmetric generalized Gaussian distributions [22]. Finally, a mapping is learned from feature space formed by the coefficients of the asymmetric gener-

125 alized Gaussian distributions and the quality scores for the LIVE Image Quality Assessment Database [23], using a support vector machine regression [24]. In 

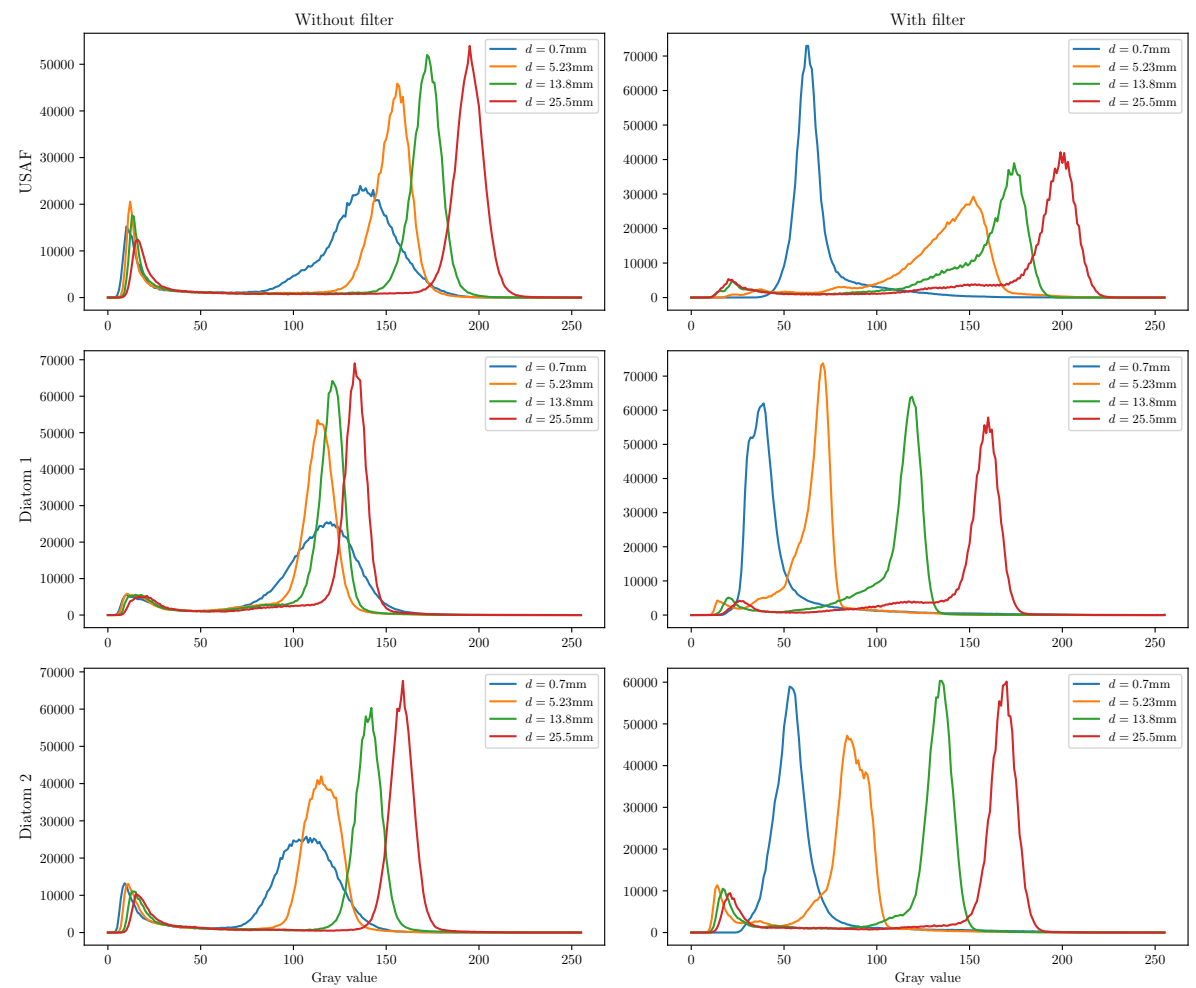

Figure 5: Histograms of the recorded images. The first column corresponds to images with the VHR filter and the second, without. USAF-1951 test target, the first and second diatom sets are represented in rows 1,2 , and 3 , respectively.

the implementation we used, available at https://github.com/gregfreeman/ image_quality_toolbox, this quality index varies between 0 and 100 being 0 an indicator of a perfect quality .

The BRISQUE scores of each set of images are shown in Fig. 6. The same trend for all the cases considered is detected: images recorded using the VHR filter produce (i) better BRISQUE scores for small values of $d$ (more coherent cases) and (ii) a minimum BRISQUE value is always detected.

\section{Concluding remarks}

In the present paper we found that the combined use of a partially coherent light source and a vortex half-wave retarder makes possible designing imaging systems able to produce gradual edge enhanced images. In the experiments, we used a conventional $4 \mathrm{f}$ imaging system with coherent-tunable illumination and a vortex half-wave retarder placed at the Fourier plane. In this way, the

final image is spirally polarized. The combination of coherence and polarization 

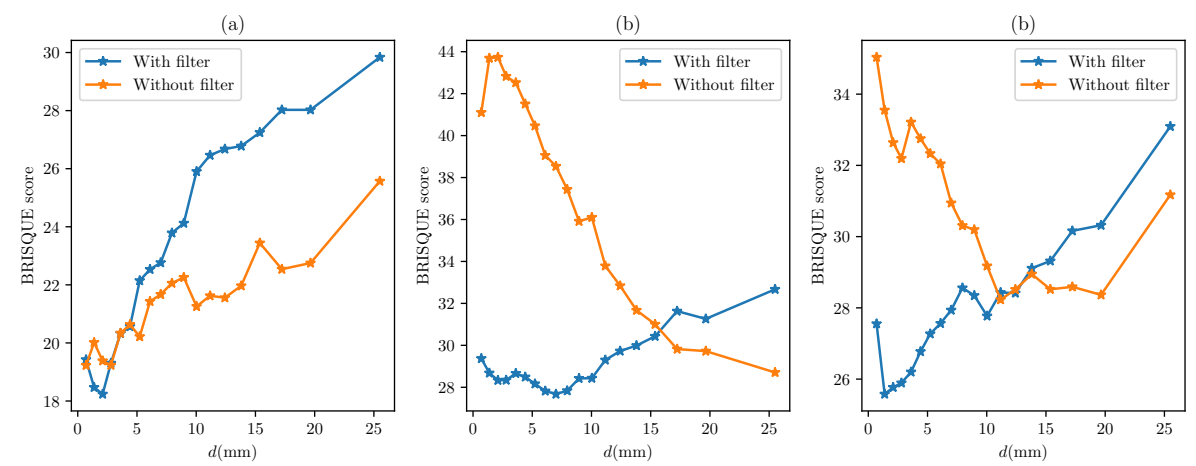

Figure 6: BRISQUE scores for each set of images. (a) USAF-1951 frequency test; (b) and (c) the two sets of diatoms. Recall that lower values represent better image quality.

effects results in a gradual edge detection and in a modification of the spatial quality content of the final image.

We assessed the quality of each image by means of the BRISQUE blind image quality estimator. We found that a minimum value of this parameter is found when using the VHR filter for a particular partially coherent state. In other words, according to BRISQUE these images present better spatial quality

\section{Acknowledgments}

RMH and AC acknowledges support from MINECO project number FIS201675147-C3-1-P.

\section{References}

[1] H. Hopkins, On the diffraction theory of optical images, Proc. Math. Phys. Eng. Sci. 217 (1130) (1953) 408-432.

[2] L. Mandel, E. Wolf, Optical coherence and quantum optics, Cambridge university press, 1995.

[3] J. W. Goodman, Statistical optics, John Wiley \& Sons, 2015.

[4] E. Wolf, Introduction to the Theory of Coherence and Polarization of Light, Cambridge University Press, 2007.

[5] R. Martínez-Herrero, P. M. Mejías, G. Piquero, Characterization of partially polarized light fields, Vol. 147, Springer Science \& Business Media, 2009 .

[6] A. T. Friberg, T. Setälä, Electromagnetic theory of optical coherence, J. Opt. Soc. Am. A 33 (12) (2016) 2431-2442. 
[20] J. W. Goodman, Introduction to Fourier optics, Roberts and Company Publishers, 2005.

[21] P. De Santis, F. Gori, G. Guattari, C. Palma, An example of a Collett-Wolf source, Opt. Commun. 29 (3) (1979) 256-260. 
[22] N.-E. Lasmar, Y. Stitou, Y. Berthoumieu, Multiscale skewed heavy tailed model for texture analysis, in: Image Processing (ICIP), 2009 16th IEEE International Conference on, IEEE, 2009, pp. 2281-2284.

[23] H. R. Sheikh, M. F. Sabir, A. C. Bovik, A statistical evaluation of recent full reference image quality assessment algorithms, IEEE Trans. Image Process. 15 (11) (2006) 3440-3451.

[24] B. Schölkopf, A. J. Smola, R. C. Williamson, P. L. Bartlett, New support vector algorithms, Neural comput. 12 (5) (2000) 1207-1245. 